\title{
Lecciones desde el campo de batalla: empleo de fentanilo transmucoso oral en personal militar, a propósito de un caso
}

\author{
R. Navarro Suay ${ }^{1}$, S. Castillejo Pérez ${ }^{1}$, M. Martínez Roldán², P. Borrego Martínez ${ }^{3}$ y E. López Soberón ${ }^{4}$ \\ ${ }^{1}$ Servicio de Anestesiología, Reanimación y Terapéutica del Dolor / Instituto Mixto de Investigación \\ Biosanitaria de la Defensa. ${ }^{2}$ Servicio de Cirugía Ortopédica y Traumatología. ${ }^{3}$ Servicio de Medicina \\ Intensiva. ${ }^{4}$ Servicio de Cardiología. Hospital Universitario Central de la Defensa "Gómez Ulla". Madrid
}

Navarro Suay R, Castillejo Pérez S, Martínez Roldán M, Borrego Martínez P, López Soberón E. Lecciones desde el campo de batalla: empleo de fentanilo transmucoso oral en personal militar, a propósito de un caso. Rev Soc Esp Dolor 2015; 22(2): 69-72.

\begin{abstract}
The improvements that have been progressively introduced in the treatment of pain in the battlefield have included increased training, research, availability of drugs and the most current techniques. One of analgesic that has been used first time in Iraq and Afghanistan conflicts has been oral transmucosal fentanyl (FTMO) generating an experience not only applicable in combat, but also in the civil and hospital environment. We describe a report of a soldier, who suffers an accident at work with chest and pelvic trauma requiring FTMO administration to achieve adequate pain control.
\end{abstract}

Key words: Oral transmucosal fentanyl. Military medicine. Pain. Trauma.

\section{RESUMEN}

Las mejoras que se han introducido progresivamente en el tratamiento del dolor en el campo de batalla han consistido en un incremento de la formación, del entrenamiento, de la investigación, de la disponibilidad de los medicamentos y de las técnicas más actuales. Uno de los analgésicos que se ha empleado por primera vez en los conflictos de Irak y Afganistán ha sido el fentanilo transmucoso oral (FTMO), generando una experiencia no sólo aplicable en combate, sino también en el medio civil y hospitalario. Se expone el caso de un militar, que tras un accidente laboral sufre un traumatismo costal y pélvico necesitando entre otras medidas, la administración de FTMO para conseguir un adecuado control del dolor.

Palabras clave: Fentanilo transmucoso oral. Medicina militar. Dolor. Traumatismo.

\section{INTRODUCCIÓN}

El fentanilo transmucoso oral (FTMO) es una reciente forma de administración de fentanilo, fácil e indoloro de administrar. Posee un perfil farmacodinámico único y aunque generalmente se emplea como alternativa efectiva para el tratamiento del dolor irruptivo $(1,2)$ también se puede administrar para el control de otro tipo de dolores, como el originado por un traumatismo $(3,4)$.

Las mejoras que progresivamente se han introducido en el tratamiento del dolor en el campo de batalla han consistido en un incremento de la formación, del entrenamiento, de la investigación, de la disponibilidad de los medicamentos y de las técnicas (5). Uno de los analgésicos que se ha empleado por primera vez en los conflictos de Irak y Afganistán ha sido el FTMO, generando una experiencia no sólo aplicable en combate, sino también en el medio civil y hospitalario $(6,7)$.

A continuación se expone el caso de un militar, que tras un accidente laboral sufre un traumatismo costal y pélvico necesitando entre otras medidas, la administración de FTMO para conseguir un adecuado control del dolor. 


\section{CASO CLÍNICO}

Se presenta el caso de un varón, de 32 años $(78 \mathrm{~kg}$, $179 \mathrm{~cm}$ ), sin alergias medicamentosas, con antecedentes de herniorrafia inguinal y sin ningún tratamiento, militar, que durante su jornada laboral sufrió un aplastamiento tras caída inesperada de una estructura metálica de aproximadamente $1.500 \mathrm{~kg}$. Fue evacuado en ambulancia terrestre con capacidad de soporte vital avanzado hasta el Hospital Universitario Central de la Defensa "Gómez Ulla" sin pérdida de consciencia y con estabilidad tanto respiratoria como hemodinámica.

En el Servicio de Urgencias se procedió a la valoración secundaria, a la extracción de una muestra sanguínea (destacó CPK $2.295 \mathrm{U} / \mathrm{l}$ y mioglobina $469 \mathrm{ng} / \mathrm{ml}$ con resto de parámetros de hemograma, bioquímica y hemostasia dentro de la normalidad), a la realización de radiografía de tórax compatible con contusión pulmonar leve sin fracturas costales de pelvis (Fig. 1) y TAC cervical (Fig. 2) y abdominopélvico en los que se evidenció fractura de la rama isquiopubiana izquierda sin desplazamiento, diástasis de $10 \mathrm{~mm}$ en la porción superior de la articulación sacroiliaca derecha con pequeña avulsión ósea del borde iliaco superomedial, y disminución del grosor de los espacios intersomáticos en L4-L5 y en L5-S1 acompañado de pequeña prominencia discal difusa en L4-L5.

Siendo diagnosticado como policontusionado, ingresó en la Unidad de Cuidados Intensivos con GCS de 15 puntos, pupilas reactivas simétricas, sin focalidad neurológica, con buena dinámica respiratoria, saturación de oxígeno dentro de la normalidad, perfundido, normotenso y normocárdico, sin signos de irritación peritoneal y ausencia de hematuria macroscópica por lo que a las 24 horas se decidió su alta a sala de hospitalización.

En planta hospitalaria el paciente evolucionó satisfactoriamente siendo reseñable la analítica sanguínea del sexto día de ingreso ya que apareció una elevación de enzimas hepáticas (GOT 134 U/l, GPT 91 U/l, BT 1,2 mg/ dl, BD $0,5 \mathrm{mg} / \mathrm{dl}$ ) que fue diagnosticado por el Servicio de Gastroenterología de probable hepatopatía aguda tóxica sin fallo hepático no acompañado de hepatomegalia ni de ictericia.

A los 15 días de sufrir el accidente y tras la mejora clínica y la recuperación de la normalidad en los valores analíticos, el paciente fue dado de alta del hospital recomendando revisiones periódicas a cargo del Servicio de Traumatología.

Durante su ingreso el enfermo presentó dolor de intensidad variable (EVA 2/10, 3/10, 7/10 y 1/10 durante su estancia en Urgencias, UCI, Sala de Hospitalización y al alta, respectivamente), de características punzantes, localizado en la región torácica derecha, pelvis derecha y glútea, no irradiado, que se incrementaba con la sedestación y disminuía en decúbito supino sin pro-

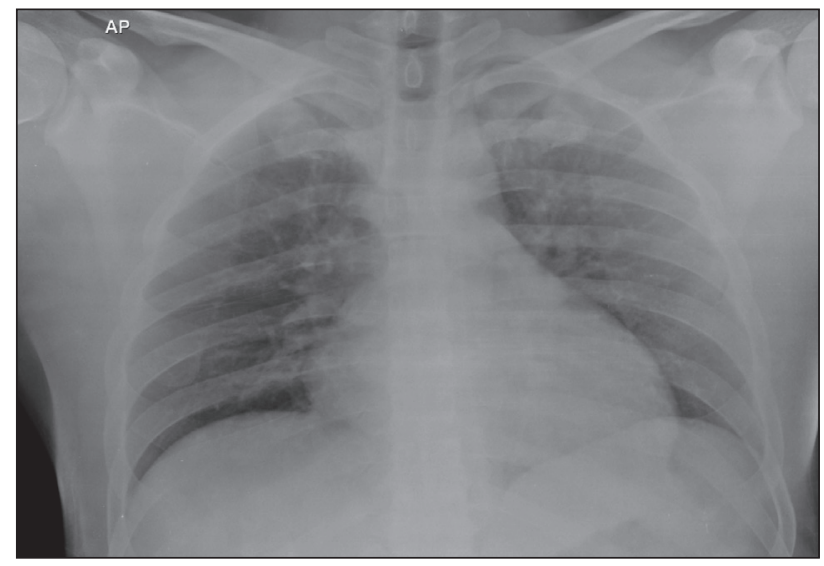

Fig. 1. Radiografía de tórax con imagen sugestiva de contusión pulmonar en ausencia de fractura costal.

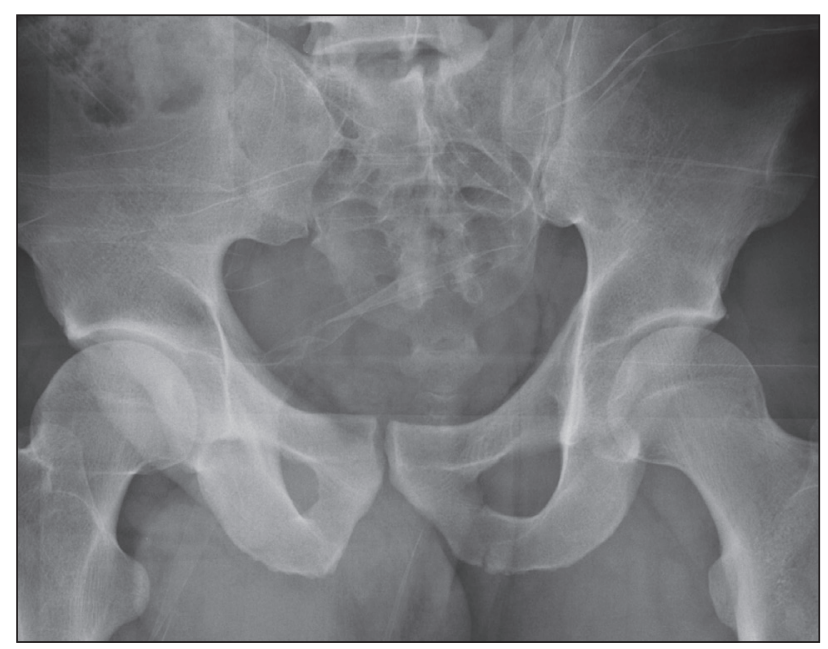

Fig. 2. Radiografía de pelvis en la que se aprecia fracturada la rama isquiopubiana izquierda sin desplazamiento.

vocar sintomatología vegetativa. Concilió el sueño de forma satisfactoria y durmió sin interrupción aproximadamente 8 horas al día. Presentó ligera distimia probablemente por no poder estar con su familia que vivía en Colombia.

El tratamiento analgésico administrado durante la evacuación hasta el hospital fue: fentanilo $100 \mu \mathrm{g}$, en la UCI: paracetamol $1 \mathrm{~g} / 8 \mathrm{~h}$ i.v. y dexketoprofenotrometamol $50 \mathrm{mg} / 8 \mathrm{~h}$ i.v., durante los 6 primeros días en sala de hospitalización: paracetamol $1 \mathrm{~g} / 8 \mathrm{~h}$ v.o. y dexketoprofeno-trometamol $50 \mathrm{mg} / 8 \mathrm{~h}$ v.o. y finalmente hasta el alta: se administró dexketoprofeno-trometamol $50 \mathrm{mg} / 8 \mathrm{~h}$ v.o. y fentanilo $400 \mu \mathrm{g}$ transmucoso 30 minutos antes de realizar cualquier movimiento potencialmente doloroso (ducharse, realización de radiografía en bipedestación...). 


\section{DISCUSIÓN}

El fentanilo se introdujo en la práctica clínica a mediados de la década de 1960. Es aproximadamente 500 veces más liposoluble que la morfina, pero con un volumen de distribución, constante de disociación del receptor $\mu \mathrm{y}$ vida media de eliminación similar a esta. La diferencia en la liposolubilidad permite un inicio de acción y una eliminación más rápidos. Cuando el FMTO se administra correctamente (frotando contra la mucosa oral durante aproximadamente 15 minutos), el $25 \%$ del medicamento se absorbe directamente, mientras que el $75 \%$ restante pasa al tubo digestivo (dos tercios se metabolizarán por un primer paso hepático y el restante originará una analgesia de inicio más lento). Conseguirá la máxima eficacia entre los 15 y 30 minutos. Como el resto de opioides el fentanilo es una agonista del receptor $\mu$, por lo que origina analgesia, ansiolisis y sedación, pudiendo provocar alucinaciones, disforia, miosis, depresión respiratoria, náuseas, vómitos, prurito y sudoración. En la actualidad se dispone de FMTO en piruletas de 200 a $1.600 \mu \mathrm{g}$, considerándose como dosis inicial única de $400 \mu \mathrm{g}$ (equivalente en potencia a 4-8 mg o $10 \mathrm{mg}$ de morfina i.v. o i.m. respectivamente) que generalmente da lugar a concentraciones plasmáticas máximas no superiores a $2 \mu \mathrm{g} / \mathrm{ml}$ (con un incremento importante de riesgo de depresión respiratoria). Debido a su perfil farmacocinético se recomienda iniciar una segunda dosis 15 minutos después de haber finalizado la dosis previa. Es importante tener en cuenta que, aunque la mediana del tiempo necesario para alcanzar la concentración plasmática máxima con la dosis de $400 \mu \mathrm{g}$ es de 25 minutos, existe un rango amplio (20-240 minutos) $(1,2,8)$. La asistencia sanitaria sobre la baja en combate procedente de los actuales conflictos asimétricos ha sufrido ciertas variaciones en cuanto al diagnóstico y al tratamiento del dolor agudo y crónico. En la actualidad, los pilares básicos de la terapia analgésica son el inicio precoz de la misma desde el lugar del incidente y a través de todos los escalones sanitarios, incorporando terapias analgésicas multimodales con el objetivo de disminuir la incidencia de dolor crónico y estrés postraumático $(7,9)$. Además, debido a las particularidades tácticas, logísticas, asistenciales y formativas del medio militar se hace necesario estandarizar el tratamiento analgésico de la baja de combate e incorporar nuevos fármacos (como en el caso del FTMO) al arsenal terapéutico de los médicos militares. Desde mediados de la década pasada varias sanidades militares -Estados Unidos (10), Gran Bretaña (8), Francia (11), Italia (12) o España (13)- emplean FTMO de manera protocolizada en bajas atendidas durante los recientes conflictos de Irak o Afganistán.

Kotwal (10) describe la analgesia conseguida en 22 heridos estadounidenses en 2003 y concluye que el FTMO puede ser una alternativa para el control del dolor en el medio militar por ser efectivo, rápido y de fácil administración al no necesitar medios invasivos para su administración. Este estudio se complementa con el de Wedmore (14), que analiza la utilización de FTMO en 286 bajas de combate norteamericanas en Iraq y Afganistán destacando la seguridad y efectividad de este fármaco en el frente. En las últimas recomendaciones del TCCC (15) se sugiere que el propio herido fije la piruleta de FTMO a su dedo mediante cinta adhesiva para que en caso de iniciarse la depresión respiratoria, con la consiguiente disminución del nivel de consciencia, se interrumpa la administración de dicho fármaco por caída involuntaria de la mano que sostenía el FTMO adherido. Asimismo Bowman (16) detalla cómo el empleo protocolizado en combate de analgésicos (entre ellos FTMO) incrementa el tratamiento precoz contra el dolor.

A nivel británico Park (17) analiza mediante una revisión sistemática la evidencia analgesia prehospitalaria civil y militar del FTMO destacando las particularidades tácticas, médicas y logísticas encontradas en el entorno bélico. De hecho, tras un proceso de validación (8) el FTMO fue elegido como parte de la estrategia multimodal a emplear por esta sanidad militar. Por su parte Davey (18) muestra cómo el $80 \%$ de los médicos militares ingleses valoraron positivamente que se incrementase la docencia sobre dicho fármaco.

Oficiales médicos españoles analizaron la analgesia administrada sobre 256 bajas en combate atendidas en el hospital militar español de Herat (Afganistán) entre 2005 y 2008. Describen que el tratamiento comenzó a nivel prehospitalario en el $61 \%$ de las bajas, el fentanilo fue administrado al $59 \%$ de los heridos y se empleó específicamente la vía transmucosa oral en 7 de ellos (13). En la actualidad de forma previa al despliegue también se instruye a los médicos militares de nuestro país para el empleo de FMTO en zona de operaciones (19).

En el caso clínico expuesto se eligió el FMTO como analgésico de rescate por sufrir el paciente un incremento del dolor (posiblemente secundario al inicio de la movilización) a pesar del tratamiento con AINE. Únicamente se empleó en un tipo específico de paciente (joven y sin enfermedades concomitantes) que sufría una situación incipiente de fallo hepático (se suspendió por este motivo el paracetamol y se eligió el FMTO por sus especiales características farmacocinéticas), exclusivamente de forma previa a la realización de ciertas maniobras potencialmente dolorosas, y aprovechando la reciente experiencia adquirida en Afganistán con bajas afectadas por patología traumática. Desde el punto de vista de los autores el resultado obtenido fue el esperado.

Así mismo, dadas las particularidades inherentes de los heridos militares (20), además del abordaje intrahospitalario de los facultativos (traumatólogos, intensivistas y anestesiólogos) creemos recomendable el apoyo por parte 
del servicio religioso, de oficiales psicólogos, de los compañeros, mandos y servicio médico de su unidad militar, así como de la recientemente creada Unidad de Apoyo a heridos y familiares de fallecidos y heridos en acto de servicio de las Fuerzas Armadas. Puede ser un modo multidisciplinar e integral de asistir a una baja militar.

\section{CONCLUSIÓN}

Una de las lecciones identificadas por los médicos militares durante los recientes conflictos de Irak y Afganistán es la necesidad de conseguir un adecuado control del dolor en la baja militar. Varias sanidades militares cuentan para ello con el FMTO desde la década pasada. La experiencia obtenida con dicho fármaco en ese medio puede emplearse igualmente en enfermos atendidos en hospitales civiles tal y como se describe en este caso clínico.

\section{CORRESPONDENCIA:}

Ricardo Navarro Suay

Servicio de Anestesiología, Reanimación y Terapéutica del Dolor

Hospital Universitario Central de la Defensa "Gómez Ulla"

Glorieta del Ejército, 1

28047 Madrid

e-mail: r_navarro_suay@yahoo.es

\section{BIBLIOGRAFÍA}

1. Shoemaker SA. Manejo del dolor irruptivo con Actiq ${ }^{\circledR}$ : aspectos prácticos. Rev Soc Esp del Dolor 2002;9:75-7.

2. Corli O, Roberto A. Pharmacological and clinical differences among transmucosal fentanyl formulations for the treatment of breakthrough cancer pain. A review article. Minerva Anestesiol 2014;80(10):1123-34.

3. Shah H, Smythe J, Hanafiah Z, Williams GJ, Holdcroft A. Factor in the choice of oral transmucosal fentanyl citrate dose for adult burns dressings. Burns 2009;35(6):798-801.

4. Mahar PJ, Rana JA, Kennedy CS, Christopher NC. A randomized clinical trial of oral transmucosal fentanyl citrate versus intravenous morphine sulfate for initial control of pain in children with extremity injuries. Pediatr Emerg Care 2007;23(8):544-8.

5. Black IH, McManus J. Tratamiento del dolor en las operaciones de combate actuales. Prehospital Emergency Care 2009;3:223-7.

6. Beekley AC, Starnes BW, Sebesta JA. Lecciones de la cirugía military moderna. En: Maier RV, editor. Abordaje actual

de los traumatismos. Barcelona: Elsevier-Masson; 2008. p. 157-84.

7. Baker BC, Buckenmaier C, Narine N, Compeggie ME, Brand GJ, Mongan PD. Anestesia en el campo de batalla: avances en la asistencia del paciente y el tratamiento del dolor. En: Shamir MY, Weiss YG, editores. Traumatismo. Barcelona: Elsevier-Masson; 2008. p. 131-45.

8. Aldington D, Jagdish S. The fentanyl "lozenge" story: From books to battlefield. J R Army Med Corps 2014. In press.

9. Cifu DX, Taylor BC, Carne WF, Bidelspach D, Sayer NA, Scholten J, e tal. Traumatic brain injury, posttraumatic stress disorder and pain diagboses in OIF/OEF/OND Veterans. J Rehabil Res Dev 2014;50(9):1169-76.

10. Kotwal RS, O’Connor KC, Johnson TR, Mosely DS, Meyer DE, Holcomb JB. A novel pain management strategy for combat casualty care. Ann Emerg Med 2004;44:121-7.

11. Fontaine B, Gentile A, Francois N, Kearns K, Cruc M, Friscth N. Analgésie du blesse de guerre au niveau du role I: Peut-on mieux faire? Carum 2010;26(2):36-7.

12. Pozza M. Confronto tra BATLS e ATLS; utilizzo dei nouvi protocolli e dispositive medici negli attuali teatri operative. Possibili applicazioni in ámbito civile. Confronto tra emergenza nel campo di battaglia con lémergenza in ámbito civile. [Tesis doctoral]. Modena: Univesitá degli studi di Modena e Reggio Emilia; 2012.

13. Navarro R, Castillejo S. Análisis de la analgesia en la baja de combate. Experiencia de la Sanidad Militar española. Sanid Mil 2012;68(1):7-15.

14. Wedmore IS, Kotwal RS, McManus JG, Pennardt A, Talbot TS, Fowler M, et al. Safety and efficacy of oral transmucosal fentanyl citrate for prehospital pain control on the battlefield. J Trauma Acute Care Surg 2012;73:490-5.

15. Butler FK, Kotwal RS, Buckenmaier CC, Edgar EP, $\mathrm{O}^{\prime}$ Connor KO, Montgomery HR, et al. A Triple-Option analgesia plan for Tactical Combat Casualty Care TCCC proposed change 13-04. Journal of Special Operations Medicine [en línea]. 2013:115-20. [20 febrero 2014]. Disponible en: https://www.jsomonline.org/TCCC/TCCC\%20 Guidelines\%20130830.pdf

16. Bowman WJ, Nesbitt ME, Therien SP. The effects of standardized trauma training on prehospital pain control: Have pain medication administration rates increased on the battlefield? J Trauma Acute Care Surg 2012;73(2):43-8.

17. Park CL, Roberts DE, Aldington DJ, Moore RA. Prehospital analgesia: Systematic review of evidence J R Army Med Corps 2010;156(4):295-300.

18. Davey CMT, Mieville KE, Simpson R, Aldington D. A proposed model for improving battlefield analgesia training: Post-graduate medical officer pain management day. J R Army Med Corps 2012;158(3):1-4.

19. Navarro Suay R. Analgesia y sedación en combate. En: Curso de Soporte Vital Avanzado en Combate. Escuela Militar de Sanidad (Madrid). 18-22 febrero 2013.

20. Clark ME, Scholten JD, Walker RL, Gironda RJ, Assessment and treatment of pain associated with combat-related polytrauma. Pain Med 2009;10(3):456-69. 